\title{
Neutrosophic number goal programming for multi-objective linear programming problem in neutrosophic number environment
}

\begin{abstract}
Purpose: The purpose of the paper is to propose goal programming strategy to multiobjective linear programming problem with neutrosophic numbers which we call NN-GP. The coefficients of objective functions and the constraints are considered as neutrosophic numbers of the form $(\mathrm{m}+\mathrm{nI})$, where $\mathrm{m}, \mathrm{n}$ are real numbers and I denotes indeterminacy.

Design: For this study, the neutrosophic numbers are converted into interval numbers. Then, the problem reduces to multi-objective linear interval programming problem. Employing interval programming technique, the target interval of the objective function is determined. For the sake of achieving the target goals, the goal achievement functions are constructed. Three new neutrosophic goal programming models are developed using deviational variables to solve the reduced problem.

Findings: Realistic optimization problem involves multiple objectives. Crisp multiobjective optimization problems involve deterministic objective functions and/or constrained functions. However, uncertainty involves in real problems. Hence, several strategies dealing with uncertain multi-objective programming problems have been proposed in the literature. Multi-objective linear programming has evolved along with different paradigms and in different environment. Goal programming and fuzzy goal programming have been widely used to solve the multi-objective linear programming problems. In this paper goal programming in neutrosophic number environment has been developed. It deals with effectively multi-objective linear programming problem with neutrosophic numbers. We solve a numerical example to illustrate the proposed NN-GP strategy.
\end{abstract}

Originality: There are different Schools in optimization field and each has their own distinct strategy. In neutrosophic number environment goal programming for multiobjective programming problem is proposed here at first.

Keywords: Neutrosophic goal programming, fuzzy goal programming, Multiobjective programming, neutrosophic numbers
Volume I Issue 3 - 2018

Surapati Pramanik,' Durga Banerjee² 'Department of Mathematics, Nandalal Ghosh B T College, India

${ }^{2}$ Department of Mathematics, Ranaghat Yusuf Institution, India

Correspondence: Surapati Pramanaik, Department of Mathematics, Nandalal Ghosh B T College, Panpur, P.O. Narayanpur, District. North 24 Parganas, PIN- 743126, West Bengal, India,Tel +919477035544, Email sura_pati@yahoo.co.in

Received: May 29, 2018 | Published: June 22, 2018

\section{Introduction}

In multi-criteria decision making (MCDM) process, multiobjective programming evolves in many directions. In multiobjective programming, several conflicting objective functions are simultaneously considered. When the objective functions and constraints both are linear, the multi-objective programming problem is considered as a linear multi-objective programming problem. If any objective function and/or constraint is nonlinear, then the problem is considered as a nonlinear multi-objective programming problem. Goal programming is a widely used strong mathematical tool to deal multi-objective mathematical programming problems. The idea of goal programming lies in the work of Chames, Cooper \& Ferguson. ${ }^{1}$ Charnes \& Cooper $^{2}$ first coined the term goal programming to deal with infeasible linear programming in 1961. GP underlies a realistic satisficing philosophy. Charnes \& Cooper, ${ }^{2}$ Ijiri, $^{3}$ Lee, ${ }^{4}$ Ignizio, ${ }^{5}$ Romero, ${ }^{6}$ Schniederjans, ${ }^{7}$ Chang, ${ }^{8}$ Dey \& Pramanik ${ }^{9}$ and many pioneer researchers established different approaches to goal programming in crisp environment. Inuguchi \& Kume ${ }^{10}$ investigated interval goal programming. Narasimhan ${ }^{11}$ grounded the goal programming using deviational variables in fuzzy environment. Fuzzy goal programming (FGP) has been enriched by several authors such as Hannan, ${ }^{12}$ Ignizio, ${ }^{13}$ Tiwari, Dharma \& Rao, ${ }^{14,15}$ Mohamed,,${ }^{16}$ Pramanik, ${ }^{17,18}$ Pramanik \& Roy, ${ }^{19-21}$ Pramanik \& Dey, ${ }^{22}$ Pramanik et al., ${ }^{23}$ Tabrizi, Shahanaghi \& Jabalameli. ${ }^{24}$ Pramanik \& Roy ${ }^{25-27}$ studied fuzzy goal programming strategy for transportation problems. Pramanik \& Roy ${ }^{28}$ presented goal programming in intuitionistic fuzzy environment, which is called intuitionistic FGP (IFGP). Pramanik \& Roy ${ }^{29}$ studied IFGP approach in transportation problems. Pramanik \& Roy $^{30}$ employed IFGP to quality control problem. Pramanik, Dey \& Roy ${ }^{31}$ studied bi-level programming problem in intuitionistic fuzzy environment. Razmi et al., ${ }^{32}$ studied Pareto-optimal solutions for intuitionistic multi-objective programming problems. Smarandache ${ }^{33}$ developed neutrosophic set based on neutrosophy. Neutrosophic set ${ }^{33}$ accommodates inconsistency, incompleteness, indeterminacy in a new angle by introducing indeterminacy as independent component. Wang, Smarandache, Zhang, et al. ${ }^{34}$ made neutrosophic theory popular by defining single valued neutrosophic set (SVNS) to deal 
with realistic problems. SVNS has been vigorously applied in different areas such as multi criteria/ attribute decision making problems ${ }^{35-53}$, conflict resolution, ${ }^{54}$ educational problem, ${ }^{55-56}$ data mining, ${ }^{57}$ social problem, ${ }^{58-59}$ etc. Smarandache ${ }^{60-61}$ defined neutrosophic number $(\mathrm{NN})$ using indeterminacy as component and established its basic properties. The $\mathrm{NN}$ is expressed in the form $\mathrm{m}+\mathrm{nI}$, where $\mathrm{m}, \mathrm{n}$ are real numbers and I represents indeterminacy. Several authors ${ }^{62-66}$ applied NNs to decision making problems. Pramanik \& Roy ${ }^{67}$ applied NNs to teacher selection problem. $\mathrm{Ye}^{68}$ developed linear programming strategy with NNs and discussed production planning problem. $\mathrm{Ye}^{69}$ developed nonlinear programming strategy in $\mathrm{NN}$ environment.

Banerjee \& Pramanik ${ }^{70}$ first studied goal programming strategy for single objective linear programming problem and developed three neutrosophic goals programming with NNs. Multi-objective linear programming problem (MOLPP) with NNs is yet to appear in the literature. To fill the gap, we present goal programming strategy for multi-objective linear programming problem with neutrosophic numbers. The coefficients of objective functions and constraints are considered as $\mathrm{NNs}$ of the form $(\mathrm{m}+\mathrm{nI})$, where $\mathrm{m}, \mathrm{n}$ are real numbers and I represents indeterminacy. The NNs are converted into interval numbers. The entire programming problem reduces to multi-objective linear interval programming problem. The target interval of the neutrosophic number function is formulated based on the technique of interval programming. Three new neutrosophic goal programming models are formulated. A numerical example is solved to illustrate the proposed NN-GP strategy. The remainder of the paper is presented as follows: Next section presents some basic discussion regarding neutrosophic set, NNs, interval numbers. Then the following section recalls interval linear programming. Then the next section devotes to formulate neutrosophic number goal programming for multi-objective linear goal programming with NNs. Then the next section presents a numerical example. Then the next section presents the conclusion and future scope of research.

\section{Some basic discussions}

Here we present some basic definitions and properties of neutrosophic numbers, interval numbers.

\section{Neutrosophic number}

An $\mathrm{NN}^{60-61}$ is denoted by $\alpha=\mathrm{m}+\mathrm{nI}$, where $\mathrm{m}, \mathrm{n}$ are real numbers and I is indeterminacy.

$$
\begin{gathered}
\alpha=m+n \text { Iwhere } I \in\left[I^{L}, I^{U}\right] \\
\alpha=\left[m+n I^{L}, m+b I^{U}\right]=\left[\alpha^{L}, \alpha^{U}\right](\text { say })
\end{gathered}
$$

Example:

Consider the NN $\alpha=5+3 \mathrm{I}$, where 5 is the determinate part and $3 \mathrm{I}$ is the indeterminate part. Suppose $\mathrm{I} \in[0.1,0.2]$, then $\alpha$ becomes an interval $\alpha=[5.3,5.6]$. Thus for a given interval of the part I, NNs are converted into interval numbers.

\section{Some basic properties of interval number}

Here some basic properties of interval analysis ${ }^{71}$ are presented as follows:

An interval is defined by an order pair

${ }_{\alpha}{ }^{\alpha} \alpha=\left[\alpha^{L}, \alpha^{U}\right]=\left\{\beta: \alpha^{L} \leq \beta \leq \alpha^{U}, \beta \in R\right\}$, where $\alpha^{L}$ and

denote the left and right limit of the interval $\alpha$ on the real line R.

Assume that $\mathrm{m}(\alpha)$ and $\mathrm{w}(\alpha)$ be the midpoint and the width respectively of an interval $\alpha$.

Then, $m(\alpha)=(1 / 2)\left(\alpha^{L}+\alpha^{U}\right)$ and $\mathrm{w}(\alpha)=\left(\alpha^{U}-\alpha^{L}\right)$

The different operations on $\alpha$ (Moore, 1966) are defined as follows:

The scalar multiplication of $\alpha$ is defined as:

$$
\lambda \alpha=\left\{\begin{array}{l}
{\left[\lambda \alpha^{L}, \lambda \alpha^{U}\right], \lambda \geq 0} \\
{\left[\lambda \alpha^{U}, \lambda \alpha^{L}\right], \lambda \leq 0}
\end{array}\right\}
$$

Absolute value of $\alpha$ is defined as

$$
|\alpha|=\left\{\begin{array}{l}
{\left[\alpha^{L}, \alpha^{U}\right], \quad \alpha^{L} \geq 0} \\
{\left[0, \max \left(-\alpha^{\mathrm{L}}, \alpha^{U}\right)\right], \quad \alpha^{L}<0<\alpha^{U}} \\
{\left[-\alpha^{U},-\alpha^{L}\right], \quad \alpha^{\mathrm{U}} \leq 0}
\end{array}\right.
$$

The binary operation '*' is defined between two interval numbers $\alpha=\left[\alpha^{L}, \alpha^{U}\right]$ and $\beta=\left[\beta^{L}, \beta^{U}\right]$ as $\alpha * \beta=\{a * b: a \in \alpha, b \in \beta\}$ where $\alpha^{L} \leq a \leq \alpha^{U}, \beta^{L} \leq b \leq \beta^{U}$.

'*' is designated as any of the operation of four conventional arithmetic operations.

\section{Some basic properties of NNs}

Here we present some properties of $\mathrm{NNs}^{60-61}$.

$$
\begin{aligned}
& \text { Let } \alpha_{1}=\mathrm{a}_{1}+\mathrm{b}_{1} \mathrm{I}_{1} \text { and } \alpha_{2}=\mathrm{a}_{2}+\mathrm{b}_{2} \mathrm{I}_{2} \text { where } \\
& \mathrm{I}_{1} \in\left[\mathrm{I}_{1}^{\mathrm{L}}, \mathrm{I}_{1}^{\mathrm{U}}\right], \mathrm{I}_{2} \in\left[\mathrm{I}_{2}^{\mathrm{L}}, \mathrm{I}_{2}^{\mathrm{U}}\right] \text { then } \\
& \qquad \alpha_{1}=\left[\mathrm{a}_{1}+\mathrm{b}_{1} \mathrm{I}_{1}^{\mathrm{L}}, \mathrm{a}_{1}+\mathrm{b}_{1} \mathrm{I}_{1}^{\mathrm{U}}\right]=\left[\alpha_{1}^{\mathrm{L}}, \alpha_{1}^{\mathrm{U}}\right] \text { (say) and } \\
& \alpha_{2}=\left[\mathrm{a}_{2}+\mathrm{b}_{2} \mathrm{I}_{2}^{\mathrm{L}}, \mathrm{a}_{2}+\mathrm{b}_{2} \mathrm{I}_{2}^{\mathrm{U}}\right]=\left[\alpha_{2}^{\mathrm{L}}, \alpha_{2}^{\mathrm{U}}\right] \text { (say). } \\
& \alpha_{1}+\alpha_{2}=\left[\alpha_{1}^{\mathrm{L}}+\alpha_{2}^{\mathrm{L}}, \alpha_{1}^{\mathrm{U}}+\alpha_{2}^{\mathrm{U}}\right] \\
& \alpha_{1}-\alpha_{2}=\left[\alpha_{1}^{\mathrm{L}}-\alpha_{2}^{\mathrm{U}}, \alpha_{1}^{\mathrm{U}}-\alpha_{2}^{\mathrm{L}}\right]
\end{aligned}
$$

$$
\begin{gathered}
\alpha_{1} * \alpha_{2}=\left[\min \left(\alpha_{1}^{\mathrm{L}} * \alpha_{2}^{\mathrm{L}}, \alpha_{1}^{\mathrm{L}} * \alpha_{2}^{\mathrm{U}}, \alpha_{1}^{\mathrm{U}} * \alpha_{2}^{\mathrm{L}}, \alpha_{1}^{\mathrm{U}} * \alpha_{2}^{\mathrm{U}}\right), \max \left(\alpha_{1}^{\mathrm{L}} * \alpha_{2}^{\mathrm{L}}, \alpha_{1}^{\mathrm{L}} * \alpha_{2}^{\mathrm{U}}, \alpha_{1}^{\mathrm{U}} * \alpha_{2}^{\mathrm{L}}, \alpha_{1}^{\mathrm{U}} * \alpha_{2}^{\mathrm{U}}\right)\right] \\
\alpha_{1} \div \alpha_{2}=\left\{\begin{array}{l}
{\left[\alpha_{1}^{\mathrm{L}}, \alpha_{1}^{\mathrm{U}}\right] *\left[\frac{1}{\alpha_{2}^{\mathrm{U}}}, \frac{1}{\alpha_{2}^{\mathrm{L}}}\right] \text { or }} \\
{\left[\min \left(\alpha_{1}^{\mathrm{L}} / \alpha_{2}^{\mathrm{L}}, \alpha_{1}^{\mathrm{L}} / \alpha_{2}^{\mathrm{U}}, \alpha_{1}^{\mathrm{U}} / \alpha_{2}^{\mathrm{L}}, \alpha_{1}^{\mathrm{U}} / \alpha_{2}^{\mathrm{U}}\right), \max \left(\alpha_{1}^{\mathrm{L}} / \alpha_{2}^{\mathrm{L}}, \alpha_{1}^{\mathrm{L}} / \alpha_{2}^{\mathrm{U}}, \alpha_{1}^{\mathrm{U}} / \alpha_{2}^{\mathrm{L}}, \alpha_{1}^{\mathrm{U}} / \alpha_{2}^{\mathrm{U}}\right)\right] \text { if } 0 \notin \alpha_{2} .} \\
\text { Undefined if } 0 \in \alpha_{2}
\end{array}\right.
\end{gathered}
$$




\section{Interval valued linear programming}

In this section, first we recall the general model of interval linear programming.

Optimize $\mathrm{C}_{\mathrm{p}}(\overline{\mathrm{Y}})=\sum_{\mathrm{j}=1}^{\mathrm{n}}\left[\mathrm{c}_{\mathrm{pj}}^{\mathrm{L}}, \mathrm{c}_{\mathrm{pj}}^{\mathrm{U}}\right] \mathrm{y}_{\mathrm{j}}, \quad \mathrm{p}=1,2, \ldots, \mathrm{P}$

$$
\begin{array}{cc}
\text { subject to } & \overline{\mathrm{A}} \overline{\mathrm{Y}}\left(\begin{array}{l}
\geq \\
= \\
\leq
\end{array}\right) \overline{\mathrm{b}} \\
\overline{\mathrm{Y}}=\left(\mathrm{y}_{1}, \mathrm{y}_{2}, \ldots, \mathrm{y}_{\mathrm{n}}\right) \geq 0
\end{array}
$$

where $\overline{\mathrm{Y}}$ is a decision vector of order $\mathrm{n} \times 1,\left[\mathrm{c}^{\mathrm{L}}, \mathrm{c}^{\mathrm{U}}\right](\mathrm{j}=1,2, \ldots$, $\mathrm{n} ; \mathrm{p}=1,2, \ldots, \mathrm{P})$ is interval coefficient of $\mathrm{p}$-th objective function, $\bar{A}$ is $\mathrm{q} \times \mathrm{n}$ matrix, $\bar{b}$ is $\mathrm{q} \times 1$ vector and $c_{p j}^{L}$ and $c_{p j}^{U}$ represent lower and upper bounds of the coefficients respectively.

Again, the multi objective linear programming with interval coefficients in objective functions as well as constraints can be presented as:

$$
\begin{array}{lll}
\text { Optimize } & \mathrm{C}_{\mathrm{p}}(\overline{\mathrm{Y}})=\sum_{\mathrm{j}=1}^{\mathrm{n}}\left[\mathrm{c}_{\mathrm{pj}}^{\mathrm{L}}, \mathrm{c}_{\mathrm{pj}}^{\mathrm{U}}\right] \mathrm{y}_{\mathrm{j}}, \quad \mathrm{p}=1,2, \ldots, \mathrm{P} \\
\text { subject to } & \sum_{\mathrm{j}=1}^{\mathrm{n}}\left[\mathrm{a}_{\mathrm{kj}}^{\mathrm{L}}, \mathrm{a}_{\mathrm{kj}}^{\mathrm{U}}\right] \mathrm{y}_{\mathrm{j}} \leq\left[\mathrm{b}_{\mathrm{k}}^{\mathrm{L}}, \mathrm{b}_{\mathrm{k}}^{\mathrm{U}}\right], \quad \mathrm{k}=1,2, \ldots, \mathrm{q}
\end{array}
$$

Here $\bar{Y}$ is a decision vector of order $\mathrm{nx} 1,\left[\mathrm{c}_{\mathrm{pj}}^{\mathrm{L}}, \mathrm{c}_{\mathrm{pj}}^{\mathrm{U}}\right],\left[\mathrm{b}_{\mathrm{k}}^{\mathrm{L}}, \mathrm{b}_{\mathrm{k}}^{\mathrm{U}}\right]$

$(j=1,2, \ldots, n ; k=1,2, \ldots, q ; p=1,2, \ldots, P)$ are closed intervals.

According to Shaocheng ${ }^{72} \&$ Ramadan $^{73}$, the interval inequality of the form

$$
\sum_{j=1}^{n}\left[a_{k j}^{\mathrm{L}}, a_{k j}^{U}\right] y_{j} \geq\left[b_{k}^{\mathrm{L}}, b_{k}^{U}\right], \quad \mathrm{k}=1,2, \ldots, \mathrm{q}
$$

$\sum_{j=1}^{n}\left[a_{j}^{L} y_{j}, a_{j}^{U} y_{j}\right] \geq\left[b^{L}, b^{U}\right] \forall y_{j} \geq 0$ can be written as the two inequalities

$$
\sum_{j=1}^{n} a_{j}^{L} y_{j} \geq b^{U} \sum_{j=1}^{n} a_{j}^{U} y_{j} \geq b^{L} \forall y_{j} \geq 0
$$

Minimization problem $^{73}$ is stated as:

Minimize $\quad \mathrm{C}_{\mathrm{p}}(\overline{\mathrm{Y}})=\sum_{\mathrm{j}=1}^{\mathrm{n}}\left[\mathrm{c}_{\mathrm{pj}}^{\mathrm{L}}, \mathrm{c}_{\mathrm{pj}}^{\mathrm{U}}\right] \mathrm{y}_{\mathrm{j}}, \quad \mathrm{p}=1,2, \ldots, \mathrm{P}$

subject to $\quad \sum_{j=1}^{\mathrm{n}}\left[\mathrm{a}_{\mathrm{kj}}^{\mathrm{L}}, \mathrm{a}_{\mathrm{kj}}^{\mathrm{U}}\right] \mathrm{y}_{\mathrm{j}} \geq\left[\mathrm{b}_{\mathrm{k}}^{\mathrm{L}}, \mathrm{b}_{\mathrm{k}}^{\mathrm{U}}\right], \quad \mathrm{k}=1,2, \ldots, \mathrm{q}$

$$
\mathrm{C}_{\mathrm{p}}(\overline{\mathrm{Y}})=\sum_{j=1}^{\mathrm{n}}\left(\mathrm{a}_{\mathrm{pj}}+\mathrm{I}_{\mathrm{pj}} \mathrm{b}_{\mathrm{pj}}\right) \mathrm{y}_{\mathrm{j}}=\sum_{\mathrm{j}=1}^{\mathrm{n}}\left[\left(\mathrm{a}_{\mathrm{pj}}+\mathrm{I}_{\mathrm{pj}}^{\mathrm{L}} \mathrm{b}_{\mathrm{pj}}\right) \mathrm{y}_{\mathrm{j}},\left(\mathrm{a}_{\mathrm{pj}}+\mathrm{I}_{\mathrm{pj}}^{\mathrm{U}} \mathrm{b}_{\mathrm{pj}}\right) \mathrm{y}_{\mathrm{j}}\right]=\left[\sum_{\mathrm{j}=1}^{\mathrm{n}}\left(\mathrm{a}_{\mathrm{pj}}+\mathrm{I}_{\mathrm{pj}}^{\mathrm{L}} \mathrm{b}_{\mathrm{pj}}\right) \mathrm{y}_{\mathrm{j}}, \sum_{\mathrm{j}=1}^{\mathrm{n}}\left(\mathrm{a}_{\mathrm{pj}}+\mathrm{I}_{\mathrm{pj}}^{\mathrm{U}} \mathrm{b}_{\mathrm{pj}}\right) \mathrm{y}_{\mathrm{j}}\right]=\left[\mathrm{C}_{\mathrm{p}}^{\mathrm{L}}, \mathrm{C}_{\mathrm{p}}^{\mathrm{U}}\right](\mathrm{say})
$$

where, $\sum_{j=1}^{n}\left(a_{\dot{p}}+I_{\dot{p}}^{\mathrm{L}} b_{\dot{p}}\right) y_{j}=C_{p}^{L}$ and $\sum_{j=1}^{n}\left(a_{\dot{p}}+I_{\dot{p}}^{U} b_{\dot{p}}\right) y_{j}=C_{p}^{U}$

The constraints reduce to

$$
\begin{aligned}
& \sum_{j=1}^{n}\left(c_{k j}+I_{k j} d_{k j}\right) y_{j} \leq \alpha_{k}+I_{k} \beta_{k} \\
& \Rightarrow\left[\sum_{j=1}^{n}\left(c_{k j}+I_{k j}^{L} d_{k j}\right) y_{j}, \sum_{j=1}^{n}\left(c_{k j}+I_{k j}^{U} d_{k j}\right) y_{j}\right] \leq\left[\alpha_{k}+I_{k}^{L} \beta_{k}, \alpha_{k}+I_{k}^{U} \beta_{k}\right] \\
& \text { Let } \alpha_{k}+I_{k}^{L} \beta_{k}=b_{k}^{L}, \alpha_{k}+I_{k}^{U} \beta_{k}=b_{k}^{U} \\
& \text { Then }\left[\sum_{j=1}^{n}\left(c_{k j}+I_{k j}^{L} d_{k j}\right) y_{j}, \sum_{j=1}^{n}\left(c_{k j}+I_{k j}^{U} d_{k j}\right) y_{j}\right] \leq\left[b_{k}^{L}, b_{k}^{U}\right], k=1,2, \ldots, q .
\end{aligned}
$$

For the best optimal solution, we solve the problem

Minimize $\quad C_{p}(\bar{Y})=\sum_{j=1}^{n} c_{p j}^{L} y_{j}, \quad p=1,2, \ldots, P$

subject to $\quad \sum_{\mathrm{j}=1}^{\mathrm{n}} \mathrm{a}_{\mathrm{kj}}^{\mathrm{U}} \mathrm{y}_{\mathrm{j}} \geq \mathrm{b}_{\mathrm{k}}^{\mathrm{L}}, \quad \mathrm{k}=1,2, \ldots, \mathrm{q}$

For the worst solution, we solve the problem

Minimize $\quad C_{p}(\bar{Y})=\sum_{j=1}^{n} c_{p j}^{U} y_{j}, \quad p=1,2, \ldots, P$

subject to $\quad \sum_{j=1}^{\mathrm{n}} \mathrm{a}_{\mathrm{kj}}^{\mathrm{L}} \mathrm{y}_{\mathrm{j}} \geq \mathrm{b}_{\mathrm{k}}^{\mathrm{U}}, \quad \mathrm{k}=1,2, \ldots, \mathrm{q}$

Suppose, the best solution point by solving (9) is

$$
\bar{Y}^{B}=\left(y_{1}^{B}, y_{2}^{B}, \ldots, y_{n}^{B}\right) \geq 0
$$

With the best objective value $\mathrm{C}^{\mathrm{B}}{ }_{\mathrm{p}}\left(\overline{\mathrm{Y}}^{\mathrm{B}}\right)=\sum_{\mathrm{j}=1}^{\mathrm{n}} \mathrm{c}_{\mathrm{pj}}^{\mathrm{L}} \mathrm{y}^{\mathrm{B}}{ }_{\mathrm{j}}, \quad \mathrm{p}=1,2, \ldots, \mathrm{P}$

Suppose, the worst solution point by solving (10) is

$$
\overline{\mathrm{Y}}^{\mathrm{W}}=\left(\mathrm{y}_{1}^{\mathrm{w}}, \mathrm{y}_{2}^{\mathrm{w}}, \ldots, \mathrm{y}^{\mathrm{w}}{ }_{\mathrm{n}}\right) \geq 0
$$

With the worst objective value

$$
\mathrm{C}^{\mathrm{W}}{ }_{\mathrm{p}}\left(\overline{\mathrm{Y}}^{\mathrm{W}}\right)=\sum_{\mathrm{j}=1}^{\mathrm{n}} \mathrm{c}_{\mathrm{pj}}^{\mathrm{L}} \mathrm{y}_{\mathrm{j}}^{\mathrm{W}}, \quad \mathrm{p}=1,2, \ldots, \mathrm{P}
$$

Then the optimal value of the $\mathrm{p}$-th objective function is

$$
\left[\mathrm{C}_{\mathrm{p}}^{\mathrm{B}}\left(\overline{\mathrm{Y}}^{\mathrm{B}}\right), \mathrm{C}_{\mathrm{p}}^{\mathrm{W}}\left(\overline{\mathrm{Y}}^{\mathrm{W}}\right)\right] \text {. }
$$

Now using the technique of goal programming we would get the optimal solution of the problem.

\section{Neutrosophic number goal programming for multi- objective linear programming problem in neutrosophic number environment}

Consider the minimization problem stated as follows:

Minimize $C_{p}(\bar{Y})=\sum_{j=1}^{n}\left(a_{p j}+I_{p j} b_{p j}\right) y_{j} \quad p=1,2, \ldots, P$

Subjected to $\sum_{j=1}^{n}\left(c_{k j}+I_{k j} d_{k j}\right) y_{j} \leq \alpha_{K}+I_{k} \beta_{k}$,

Where $I_{p j} \in\left[I_{p j}^{L}, I_{p j}^{U}\right]$ and $I_{k j} \in\left[I_{k j}^{L}, I_{k j}^{U}\right] I_{k} \in\left[I_{k}^{L}, I_{k}^{U}\right] j=1,2, \ldots \ldots .$, $\mathrm{n}$ and $\mathrm{k}=1,2, \ldots \ldots \ldots \mathrm{q}$

Now,

Assume that the decision maker fixes $\left[\mathrm{C}_{\mathrm{p}}^{*}, \mathrm{C}_{\mathrm{p}}^{* \mathrm{U}}\right]$ as the target interval of the p-th objective function.

Applying the procedure discussed in the section 3, we find out the target level of each objective function. The $\mathrm{p}$-th objective function with target is written as:

$\mathrm{C}_{\mathrm{p}}^{\mathrm{U}} \geq \mathrm{C}_{\mathrm{p}}^{* \mathrm{~L}}$ and $\mathrm{C}_{\mathrm{p}}^{\mathrm{L}} \leq \mathrm{C}_{\mathrm{p}}^{* \mathrm{U}}$

The goal achievement functions are written as:

$-\mathrm{C}_{\mathrm{p}}^{\mathrm{U}}+\mathrm{d}_{\mathrm{p}}^{\mathrm{U}}=-\mathrm{C}_{\mathrm{p}}^{* \mathrm{~L}}$ and $\mathrm{C}_{\mathrm{p}}^{\mathrm{L}}+\mathrm{d}_{\mathrm{p}}^{\mathrm{L}}=\mathrm{C}_{\mathrm{p}}^{* \mathrm{U}}$

Here $d_{p}^{L} \geq 0$, and $d_{p}^{U} \geq 0$ are negative deviational variables. 
Goal programming model I (22)

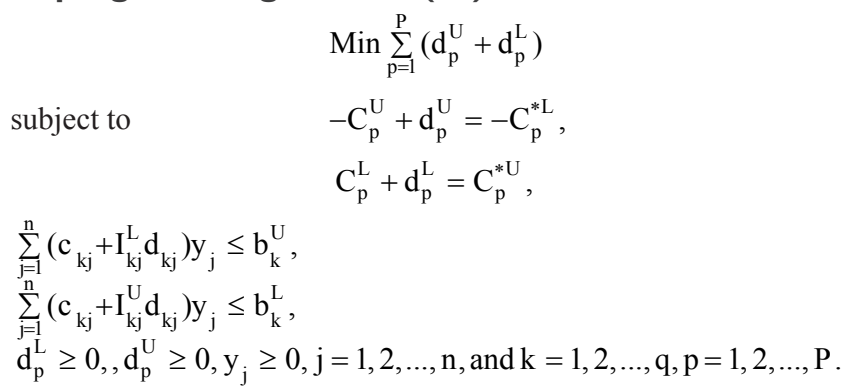

\section{Goal programming model II (23)}

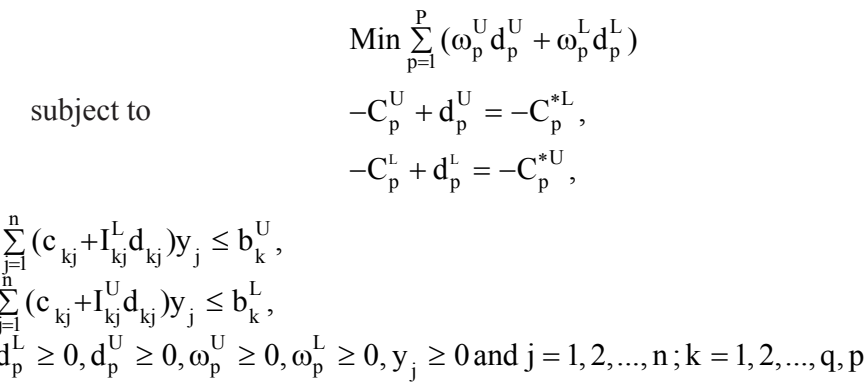

Here $\omega_{\mathrm{p}}^{\mathrm{U}}, \omega_{\mathrm{p}}^{\mathrm{L}}$ are the numerical weights of corresponding negative deviational variables suggested by decision makers.

\section{Goal programming model III (24)}

$\operatorname{Min} \lambda$

subject to $\quad-C_{\mathrm{p}}^{\mathrm{U}}+\mathrm{d}_{\mathrm{p}}^{\mathrm{U}}=-\mathrm{C}_{\mathrm{p}}^{* \mathrm{~L}}$,

$$
-\mathrm{C}_{\mathrm{p}}^{\mathrm{L}}+\mathrm{d}_{\mathrm{p}}^{\mathrm{L}}=-\mathrm{C}_{\mathrm{p}}^{* \mathrm{U}} \text {, }
$$

$$
\begin{aligned}
& \sum_{j=1}^{\mathrm{n}}\left(\mathrm{c}_{k j}+\mathrm{I}_{\mathrm{kj}}^{\mathrm{L}} \mathrm{d}_{\mathrm{kj}}\right) \mathrm{y}_{\mathrm{j}} \leq \mathrm{b}_{\mathrm{k}}^{\mathrm{U}}, \\
& \sum_{\mathrm{j}=1}^{\mathrm{n}}\left(\mathrm{c}_{\mathrm{kj}}+\mathrm{I}_{\mathrm{kj}}^{\mathrm{U}} \mathrm{d}_{\mathrm{kj}}\right) \mathrm{y}_{\mathrm{j}} \leq \mathrm{b}_{\mathrm{k}}^{\mathrm{L}}, \\
& \lambda \geq \mathrm{d}_{\mathrm{p}}^{\mathrm{U}}, \\
& \lambda \geq \mathrm{d}_{\mathrm{p}}^{\mathrm{L}},
\end{aligned}
$$

$d_{p}^{L} \geq 0, d_{p}^{U} \geq 0, y_{j} \geq 0, j=1,2, \ldots, n$, and $k=1,2, \ldots, q, p=1,2, \ldots, P$.

\section{Numerical example}

Consider the following MOLPP with NNs with I $[0,1]$.

$$
\begin{gathered}
\operatorname{Min}_{1}=(2+I) y_{1}+(4+I) y_{2} \\
\operatorname{Min}_{2}=(3+I) y_{1}+(2+I) y_{2} \\
(3+I) y_{1}+(2+4 I) y_{2} \geq(4+30 I), \\
(4+I) y_{1}+(16+I) y_{2} \geq 16, \\
y_{1} \geq 0 ; y_{2} \geq 0, I \in[0,1] .
\end{gathered}
$$

Subject to

The objective functions and the constraints reduce to the following structures:

$$
\begin{gathered}
\operatorname{Min}_{1}=\left[2 \mathrm{y}_{1}+4 \mathrm{y}_{2}, 3 \mathrm{y}_{1}+5 \mathrm{y}_{2}\right] \\
\operatorname{MinC}_{2}=\left[3 \mathrm{y}_{1}+2 \mathrm{y}_{2}, 4 \mathrm{y}_{1}+3 \mathrm{y}_{2}\right] \\
{\left[3 \mathrm{y}_{1}+2 \mathrm{y}_{2}, 4 \mathrm{y}_{1}+6 \mathrm{y}_{2}\right] \geq[4,34],} \\
{\left[4 \mathrm{y}_{1}+16 \mathrm{y}_{2}, 5 \mathrm{y}_{1}+17 \mathrm{y}_{2}\right] \geq 16,} \\
\mathrm{y}_{1} \geq 0 ; \mathrm{y}_{2} \geq 0 .
\end{gathered}
$$

The reduced problems are shown in Table 1.

The best and worst solutions are presented in Table 2 .

Table I Reduced problem

\begin{tabular}{lll}
\hline Objective function & Problem for the best solution & Problem for the worst solution \\
$C_{1}$ & $\operatorname{MinC}_{1}^{\mathrm{L}}=2 \mathrm{y}_{1}+4 \mathrm{y}_{2}$ & $\operatorname{MinC}_{1}^{\mathrm{U}}=3 \mathrm{y}_{1}+5 \mathrm{y}_{2}$ \\
& $4 \mathrm{y}_{1}+6 \mathrm{y}_{2} \geq 4 ; 5 \mathrm{y}_{1}+17 \mathrm{y}_{2} \geq 16 ;$ & $3 \mathrm{y}_{1}+2 \mathrm{y}_{2} \geq 34 ; 4 \mathrm{y}_{1}+16 \mathrm{y}_{2} \geq 16 ;$ \\
& $\mathrm{y}_{1} \geq 0 ; \mathrm{y}_{2} \geq 0$. & $\mathrm{y}_{1} \geq 0 ; \mathrm{y}_{2} \geq 0$. \\
& $\operatorname{MinC}_{2}^{\mathrm{L}}=3 \mathrm{y}_{1}+2 \mathrm{y}_{2}$ & $\operatorname{MinC}_{2}^{\mathrm{U}}=4 \mathrm{y}_{1}+3 \mathrm{y}_{2}$ \\
$C_{2}$ & $4 \mathrm{y}_{1}+6 \mathrm{y}_{2} \geq 4 ; 5 \mathrm{y}_{1}+17 \mathrm{y}_{2} \geq 16 ;$ & $3 \mathrm{y}_{1}+2 \mathrm{y}_{2} \geq 34 ; 4 \mathrm{y}_{1}+16 \mathrm{y}_{2} \geq 16 ;$ \\
& $\mathrm{y}_{1} \geq 0 ; \mathrm{y}_{2} \geq 0$. & $\mathrm{y}_{1} \geq 0 ; \mathrm{y}_{2} \geq 0$. \\
\hline
\end{tabular}

Table 2 Best and Worst solutions

\begin{tabular}{lll}
\hline Objective function & Best Solution with solution point & Worst solution with solution point \\
\hline$C_{1}$ & Min $C_{1}^{\mathrm{L}^{*}}=3.765$ & ${\operatorname{Min~} \mathrm{C}_{1}^{\mathrm{U}^{*}}=34}$ \\
at $(0,0.94 \mathrm{I})$ & at $(\mathrm{II} .333,0)$ \\
$\mathrm{C}_{2}$ & $\operatorname{Min} \mathrm{C}_{2}^{\mathrm{L}^{*}}=1.882$ & $\operatorname{Min~}_{2}^{\mathrm{U}^{*}}=45.333$ \\
& at $(0,0.94 \mathrm{I})$ & at $(\mathrm{II} .333,0)$ \\
\hline
\end{tabular}

The objective functions with targets can be written as:

$2 \mathrm{y}_{1}+4 \mathrm{y}_{2} \leq 34,3 \mathrm{y}_{1}+5 \mathrm{y}_{2} \geq 4,3 \mathrm{y}_{1}+2 \mathrm{y}_{2} \leq 46,4 \mathrm{y}_{1}+3 \mathrm{y}_{2} \geq 2$

The goal functions with targets can be written as:

$$
\begin{aligned}
& 2 \mathrm{y}_{1}+4 \mathrm{y}_{2}+\mathrm{d}_{1}^{\mathrm{L}}=34, \\
& -3 \mathrm{y}_{1}-5 \mathrm{y}_{2}+\mathrm{d}_{1}^{\mathrm{U}}=-4, \\
& 3 \mathrm{y}_{1}+2 \mathrm{y}_{2}+\mathrm{d}_{2}^{\mathrm{L}}=46,
\end{aligned}
$$




$$
\begin{aligned}
& -4 y_{1}-3 y_{2}+d_{2}^{U}=-2, \\
& d_{1}^{U} \geq 0, d_{1}^{L^{2}} \geq 0, d_{2}^{U} \geq 0, d_{2}^{L} \geq 0 .
\end{aligned}
$$

Using the goal programming model (22), the goal programming model I is presented as follows:

\section{GP Model I}

$$
\begin{gathered}
\operatorname{Min} \sum_{p=1}^{2}\left(d_{p}^{U}+d_{p}^{L}\right) \\
2 y_{1}+4 y_{2}+d_{1}^{L}=34, \\
-3 y_{1}-5 y_{2}+d_{1}^{U}=-4, \\
3 y_{1}+2 y_{2}+d_{2}^{L}=46, \\
-4 y_{1}-3 y_{2}+d_{2}^{U}=-2, \\
4 y_{1}+6 y_{2} \geq 4, \\
5 y_{1}+17 y_{2} \geq 16, \\
3 y_{1}+2 y_{2} \geq 34, \\
4 y_{1}+16 y_{2} \geq 16, \\
d_{1}^{U} \geq 0, d_{1}^{L} \geq 0, d_{2}^{U} \geq 0, d_{2}^{L} \geq 0 \\
y_{1} \geq 0 ; y_{2} \geq 0
\end{gathered}
$$

Using the goal programming model (23), the goal programming model II is presented as follows:

\section{GP Model II}

$$
\begin{gathered}
\operatorname{Min} \sum_{p=1}^{2}\left(\omega_{p}^{U} d_{p}^{U}+\omega_{p}^{L} d_{p}^{L}\right) \\
2 y_{1}+4 y_{2}+d_{1}^{L}=34, \\
-3 y_{1}-5 y_{2}+d_{1}^{U}=-4, \\
3 y_{1}+2 y_{2}+d_{2}^{L}=46, \\
-4 y_{1}-3 y_{2}+d_{2}^{U}=-2, \\
4 y_{1}+6 y_{2} \geq 4 \\
5 y_{1}+17 y_{2} \geq 16 \\
3 y_{1}+2 y_{2} \geq 34 \\
4 y_{1}+16 y_{2} \geq 16 \\
d_{1}^{U} \geq 0, d_{1}^{L} \geq 0, d_{2}^{U} \geq 0, d_{2}^{L} \geq 0 \\
y_{1} \geq 0, y_{2} \geq 0 \\
\omega_{p}^{U}, \omega_{p}^{L} \geq 0, p=1,2 .
\end{gathered}
$$

Using the goal programming model (24), the goal programming model III is presented as follows:

\section{GP Model III}

$\operatorname{Min} \lambda$

$$
\begin{aligned}
& 2 \mathrm{y}_{1}+4 \mathrm{y}_{2}+\mathrm{d}_{1}^{\mathrm{L}}=34, \\
& -3 \mathrm{y}_{1}-5 \mathrm{y}_{2}+\mathrm{d}_{1}^{\mathrm{U}}=-4, \\
& 3 \mathrm{y}_{1}+2 \mathrm{y}_{2}+\mathrm{d}_{2}^{\mathrm{L}}=46, \\
& -4 \mathrm{y}_{1}-3 \mathrm{y}_{2}+\mathrm{d}_{2}^{\mathrm{U}}=-2,
\end{aligned}
$$

$$
\begin{gathered}
4 \mathrm{y}_{1}+6 \mathrm{y}_{2} \geq 4, \\
5 \mathrm{y}_{1}+17 \mathrm{y}_{2} \geq 16, \\
3 \mathrm{y}_{1}+2 \mathrm{y}_{2} \geq 34, \\
4 \mathrm{y}_{1}+16 \mathrm{y}_{2} \geq 16, \\
\mathrm{~d}_{1}^{\mathrm{U}} \geq 0, \mathrm{~d}_{1}^{\mathrm{L}} \geq 0, \mathrm{~d}_{2}^{\mathrm{U}} \geq 0, \mathrm{~d}_{2}^{\mathrm{L}} \geq 0, \\
\mathrm{y}_{1} \geq 0, \mathrm{y}_{2} \geq 0, \\
\lambda \geq \mathrm{d}_{1}^{\mathrm{U}}, \lambda \geq \mathrm{d}_{1}^{\mathrm{L}}, \\
\lambda \geq \mathrm{d}_{2}^{\mathrm{U}}, \lambda \geq \mathrm{d}_{2}^{\mathrm{L}} .
\end{gathered}
$$

The optimal solutions are presented in Table 3 .

Table 3 Optimal solution

\begin{tabular}{llll}
\hline Programming model & $\mathbf{C}_{\mathbf{1}}$ & $\mathbf{C}_{2}$ & $\overline{\mathrm{Y}}^{*}$ \\
\hline Goal programming Model I & {$[22.67,34]$} & {$[34,45.33]$} & $($ I I.33, 0) \\
Goal programming Model II & {$[22.67,34]$} & {$[34,45.33]$} & $($ II.33,0) \\
Goal programming Model III & {$[22.67,34]$} & {$[34,45.33]$} & $($ II.33, 0) \\
\hline
\end{tabular}

\section{Conclusion}

This paper has presented the solution strategy of multi-objective linear goal programming problem with neutrosophic coefficients of both objective functions and constraints. The neutrosophic coefficients of the form $\mathrm{m}+\mathrm{nI}$ is converted into interval coefficient with the prescribed range of I. Adopting the concept of solving linear interval programming problem, three new neutrosophic goal programming models have been developed and solved by considering a numerical example. We hope that the proposed method for solving multiobjective linear goal programming with neutrosophic coefficients will lighten up a new way for the future research work. The proposed NN-GP strategy can be extended to multi-objective priority based goal programming with NNs. In future, we shall apply the proposed NN-GP strategies to production planning in brickfield, ${ }^{74}$ bi-level programming problem ${ }^{75}$ and health care management. ${ }^{76}$

\section{Acknowledgements}

None

\section{Conflict of interests}

The author declares that there is no conflict of interest.

\section{References}

1. Charnes A, Cooper WW, Ferguson A. Optimal estimation of executive compensation by linear programming. Management Science. $1955 ; 1: 138-151$

2. Charnes A, Cooper WW. Management models and industrial applications of linear programming I and II. New York: Wiley; 1961.

3. Ijiri Y. Management goals and accounting for control. North-Holland Publication: Amsterdam; 1965.

4. Lee SM. Goal programming for decision analysis. Philadelphia: Auerbach Publishers; 1972.

5. Ignizio JP. Goal programming and extensions. Lexington, Massachusetts: D. C. Health; 1976 
6. Romero C. Handbook of critical issues in goal programming Pergamon Press: Oxford; 1991.

7. Schniederjans MJ. Goal programming: Methodology and applications: methodology and applications. Kluwer Academic Publishers: Boston; 1995.

8. Chang CT. Multi-choice goal programming. Omega 2007;35(4):389396.

9. Dey PP, Pramanik, S. Goal programming approach to linear fractional bilevel programming problem based on Taylor series approximation Int J Pure and Applied Sci Technol. 2011;(2):115-123.

10. Inuiguchi M, Kume Y. Goal programming problems with interval coefficients and target intervals. European Journal Operational Research. 1991;52(3):345-361.

11. Narasimhan R. Goal programming in a fuzzy environment. Decision Sciences. 1980;11(2):325-336.

12. Hannan E L. On fuzzy goal programming. Decision Sciences. 1981;12 (3):522-531.

13. Ignizio JP. On the re discovery of fuzzy goal programming. Decision Sciences. 1982;13(2):331-336.

14. Tiwari RN, Dharma S, Rao JR. Priority structure in fuzzy goal programming. Fuzzy Sets and Systems. 1986;19(3):251-259.

15. Tiwari RN, Dharma S, Rao JR. Fuzzy goal programming - an additive model. Fuzzy Sets and Systems. 1987;24(1):27-34.

16. Mohamed RH. The relationship between goal programming and fuzzy programming. Fuzzy Sets and Systems. 1997; 89(2):215-222.

17. Pramanik S. Bilevel programming problem with fuzzy parameters: a fuzzy goal programing approach. Journal of Applied Quantitative Methods. 2012;7(1):09-24.

18. Pramanik S. Multilevel programming problems with fuzzy parameters: a fuzzy goal programming approach. International Journal of Computer Applications. 2015;122(21):34-41.

19. Pramanik S, Roy TK. A fuzzy goal programming approach for multiobjective capacitated transportation problem. Tamsui Oxford Journal of Management Sciences. 2005:21(1):75-88.

20. Pramanik S, Roy TK. A goal programming procedure for solving unbalanced transportation problem having multiple fuzzy goals. Tamsui Oxford Journal of Management Sciences. 2005;21(2):37-52.

21. Pramanik S, Roy TK. A fuzzy goal programming approach for multilevel programming problems. European Journal of Operational Research. 2007;176(2):1151-1166.

22. Pramanik S, Dey PP. Quadratic bi-level programming problem based on fuzzy goal programming approach. International Journal of Software Engineering \& Applications. 2011;2(4):41-59.

23. Pramanik S, Maiti I, Mandal T. A Taylor series based fuzzy mathematical approach for multi objective linear fractional programming problem with fuzzy parameters. International Journal of Computer Applications. 2018;180(45):22-29.

24. Tabrizi BB, Shahanaghi K, Jabalameli MS. Fuzzy multi-choice goa programming. Applied Mathematical Modelling. 2012;36(4):14151420

25. Pramanik S, Roy TK. A fuzzy goal programming technique for solving multi-objective transportation problem. Tamsui Oxford Journal of Management Sciences. 2006;22(1):67-89.

26. Pramanik S, Roy TK. Multiobjective transportation model with fuzzy parameters: a priority based fuzzy goal programming. Journal of Transportation Systems Engineering and Information Technology. 2008;8(3):40-48.
27. Pramanik S, Banerjee D. Multi-objective chance constrained capacitated transportation problem based on fuzzy goal programming. International Journal of Computer Applications. 2012;44(20):42-46.

28. Pramanik S, Roy TK. An intuitionistic fuzzy goal programming approach to vector optimization problem. Notes on Intuitionistic Fuzzy Sets. 2005;11(5):01-14.

29. Pramanik S, Roy TK. Intuitionist fuzzy goal programming and its application in solving multi-objective transportation problem. Tamsui Oxford Journal of Management Sciences. 2007;23(1):1-17.

30. Pramanik S, Roy TK. An intuitionistic fuzzy goal programming approach for a quality control problem: a case study. Tamsui Oxford Journal of Management Sciences. 2007;23(3):1-18.

31. Pramanik S, Dey, PP, Roy, TK. Bilevel programming in an intuitionistic fuzzy environment. Journal of Technology. 2011;XXXXII:103-114.

32. Razmi J, Jafarian E, Amin SH. An intuitionistic fuzzy goal programming approach for finding Pareto-optimal solutions to multiobjective programming problems. Expert Systems with Applications. 2016; 65:181-193.

33. Smarandache F. Neutrosophy: neutrosophic probability, set, and logic. American Research Press: Rehoboth; 1998.

34. Wang H, Smarandache F, Zhang YQ, et al. Single valued neutrosophic sets. Multi-space and Multi-structure 2010;4 410-413.

35. Biswas P, Pramanik S, Giri, BC. Entropy based grey relational analysis method for multi-attribute decision making under single valued neutrosophic assessments. Neutrosophic Sets and Systems. 2014;2:102-110.

36. Biswas P, Pramanik S, Giri, BC. A new methodology for neutrosophic multi-attribute decision-making with unknown weight information Neutrosophic Sets and Systems. 2014;3:44-54.

37. Biswas P, Pramanik S, Giri, BC. Cosine similarity measure based multi-attribute decision-making with trapezoidal fuzzy neutrosophic numbers. Neutrosophic Sets and Systems. 2015;8:46-56.

38. Biswas P, Pramanik S, Giri, BC. TOPSIS method for multi-attribute group decision making under single-valued neutrosophic environment. Neural Computing and Applications. 2016;27(3):727-737.

39. Biswas P, Pramanik S, Giri, BC. Value and ambiguity index based ranking method of single-valued trapezoidal neutrosophic numbers and its application to multi-attribute decision making. Neutrosophic Sets and Systems. 2016;12:127-138.

40. Broumi S, Smarandache F. Single valued neutrosophic trapezoid linguistic aggregation operators based multi-attribute decision making. Bulletin of Pure \& Applied Sciences Mathematics and Statistics, 2014;33e(2):135-155

41. Kharal A. A neutrosophic multi-criteria decision making method. New Mathematics and Natural Computation. 2014;10(2):143-162.

42. Mondal K, Pramanik S. Neutrosophic tangent similarity measure and its application to multiple attribute decision making. Neutrosophic Sets and Systems. 2015;9:80-87.

43. Mondal K, Pramanik S. Neutrosophic decision making model for claybrick selection in construction field based on grey relational analysis. Neutrosophic Sets and Systems. 2015;9:64-71.

44. Mondal K, Pramanik S, Giri BC. Single valued neutrosophic hyperbolic sine similarity measure based MADM strategy. Neutrosophic Sets and Systems. 2018;20:3-11.

45. Mondal K, Pramanik S, Giri BC. Hybrid binary logarithm similarity measure for MAGDM problems under SVNS assessments. Neutrosophic Sets and Systems. 2018;20:12-25. 
46. Pramanik S, Biswas P, Giri BC. Hybrid vector similarity measures and their applications to multi-attribute decision making under neutrosophic environment. Neural Computing and Applications. 2017;28 (5):1163-1176.

47. Pramanik S, Dalapati S, Alam, S, Smarandache S, Roy TK. NScross entropy based MAGDM under single valued neutrosophic set environment. Information. 2018;9(2):37.

48. Pramanik S, Dalapati S, Roy TK. Logistics center location selection approach based on neutrosophic multi-criteria decision making. In $\mathrm{F}$ Smarandache, S Pramanik, editors. New trends in neutrosophic theory and applications. Pons Editions: Brussels; 2016:161-174.

49. Pramanik S, Dalapati S, Roy TK. Neutrosophic multi-attribute group decision making strategy for logistics center location selection. In F. Smarandache, M. Abdel Basset, V Chang, editors. Neutrosophic operational research, Volume III (pp. 13-2). Pons Publishing House Pons asbl; Bruxelles: 2018.

50. Biswas P. Multi-attribute decision making in neutrosophic environment. Jadavpur University: Kolkata; 2018.

51. Smarandache F, Pramanik S. New trends in neutrosophic theory and applications, Brussels: Pons Editions; 2018:2.

52. Smarandache F, Pramanik S. New trends in neutrosophic theory and applications. Brussels: Pons Editions;2016.

53. Broumi S, Bakali A, Talea. Neutrosophic sets: An overview. In F. Smarandache, \& S. Pramanik (Eds., vol.2), New trends in neutrosophic theory and applications. Brussels: Pons Editions; 2018:403-434.

54. Pramanik S, Roy TK. Neutrosophic game theoretic approach to IndoPak conflict over Jammu-Kashmir. Neutrosophic Sets and Systems. 2014;2:82-101

55. Mondal K, Pramanik S. Multi-criteria group decision making approach for teacher recruitment in higher education under simplified neutrosophic environment. Neutrosophic Sets and Systems. 2014;6:2834.

56. Mondal K, Pramanik S. Neutrosophic decision making model of school choice. Neutrosophic Sets and Systems. 2015;7:62-68.

57. Mondal K, Pramanik S, Smarandache F. Role of neutrosophic logic in data mining. In F. Smarandache, \& S. Pramanik (Eds), New trends in neutrosophic theory and application. Belgium: Pons Editions; 2016:15-23.

58. Pramanik S, Chackrabarti SN. A study on problems of construction workers in West Bengal based on neutrosophic cognitive maps. International Journal of Innovative Research in Science, Engineering and Technology. 2013;2(11):6387-6394.

59. Mondal K, Pramanik S. A study on problems of Hijras in West Benga based on neutrosophic cognitive maps. Neutrosophic Sets and Systems. 2014;5:2-26.

60. Smarandache F. Introduction to neutrosophic measure, neutrosophic integral, and neutrosophic probability. Sitech and Education Publisher: Craiova; 2013.

61. Smarandache F, Introduction to neutrosophic statistics. Sitech and Education Publisher: Craiova; 2014.
62. Ye J. Multiple-attribute group decision-making method under a neutrosophic number environment. Journal of Intelligent Systems. 2016;25(3):377-386

63. Ye J. Bidirectional projection method for multiple attributes group decision making with neutrosophic numbers. Neural Computing and Applications. 2016;28(5):1021-1029.

64. Liu P, Liu X. The neutrosophic number generalized weighted power averaging operator and its application in multiple attribute group decision making. International Journal of Machine Learning and Cybernetics. 2018;9(2):347-358.

65. Zheng E, Teng F, Liu P. Multiple attribute group decisionmaking method based on neutrosophic number generalized hybrid weighted averaging operator. Neural Computing and Applications. 2017;28(8):2063-2074.

66. Mondal K, Pramanik S, Giri BC, et al. NN-harmonic mean aggregation operators-based MCGDM strategy in a neutrosophic number environment. Axioms. 2018;7(1):12.

67. Pramanik S, Roy R, Roy TK. Teacher selection strategy based on bidirectional projection measure in neutrosophic number environment. In F. Smarandache, MAbdel Basset, I El Henawy, editors. Neutrosophic operational research. Bruxelles: Pons Publishing House / Pons asbl; 2017;2:29-53.

68. Ye J. Neutrosophic number linear programming method and its application under neutrosophic number environments. Soft Computing. 2017.

69. Ye J, Cui W, Lu Z. Neutrosophic number nonlinear programming problems and their general solution methods under neutrosophic number environments. Axioms. 2018;7(1):13

70. Pramanik S, Banerjee D. Single-objective linear goal programming problem with neutrosophic numbers. International Journal of Engineering Science \& Research Technology. 2018;7(5):454-469.

71. Moore RE. Interval analysis. New Jersey: Prentice Hall;1966

72. Shaocheng T. Interval number and fuzzy number linear programming. Fuzzy Sets and Systems. 1994;66(3):301-306.

73. Ramadan K, Linear programming with interval coefficients. Carleton University: Doctoral dissertation; 1996.

74. Banerjee D, Mondal, K, Pramanik S. Fuzzy goal programming approach for soil allocation problem in brick-fields-a case study. Global Journal of Engineering Science and Research Management. 2016;3(3):1-116.

75. Dey PP, Pramanik S. Goal programming approach to linear fractional bilevel programming problem based on Taylor series approximation. International Journal of Pure and Applied Sciences and Technology. 2011;6(2):115-123.

76. Azcarate C, Mallor F, Gafaro A. Multiobjective optimization in health care management. $A$ metaheuristic and simulation approach. Algorithmic Operations Research. 2008;3(2):186-202. 